\title{
Capacité d'ingestion comparée de génisses et de vaches de race Holstein, Montbéliarde et Tarentaise *
}

\author{
JB Coulon, P D'Hour \\ INRA, laboratoire Adaptation des herbivores aux milieux, Theix, \\ 63122 Saint-Genès Champanelle, France
}

(Reçu le 4 octobre 1993; accepté le 14 mars 1994)

\begin{abstract}
Résumé - La capacité d'ingestion de génisses et de vaches de races Holstein, Montbéliarde et Tarentaise a été comparée à partir de données issues de 38 lots de 4 à 15 individus, recevant, au cours de périodes de 2 mois, des rations à base de foin offert à volonté. Au cours de chaque comparaison, les quantités ingérées observées chez les animaux Holstein ont servi à calculer la valeur d'encombrement du fourrage utilisé. En utilisant ensuite les modèles français de prévision de la capacité d'ingestion des vaches laitières et décrivant la substitution entre fourrages et concentrés, nous avons calculé les quantités ingérées prédites pour les autres types d'animaux qui ont été comparées aux valeurs observées. Les génisses et les vaches Montbéliardes ont présenté une capacité d'ingestion très voisine de celle des animaux Holstein, de même que les génisses et les vaches Tarentaises primipares. Les vaches Tarentaises multipares ont présenté une capacité d'ingestion inférieure d'environ 1 UEL à celle prédite par le modèle. Les causes possibles de cet écart sont discutées, en particulier celles liées à l'état corporel des animaux.
\end{abstract}

ingestion / génisse / vache laitière / race

Summary - Feed intake capacity in heifers and cows of different breeds. Feed intake capacity was compared in heifers and cows of different breeds (Holstein, Montbéliarde and Tarentaise) using data from 38 groups of 4-15 animals fed with hay (ad libitum) and concentrates (depending on their requirements) during periods of 2 months. During each comparison, data from the Holstein group were used to calculate the ingestibility of the roughage used. Using the predictive models of feed intake capacity and substitution rate, developed in the French Fill Unit systems, predicted feed intake of the other breeds were calculated and compared with observed data. Montbéliarde heifers and cows, Tarentaise heifers and Tarentaise primiparous cows had the same feed intake capacity than Holstein animals. Tarentaise multiparous cows had a feed intake capacity lower than Holstein multiparous cows (about -7\%). The possible origins of this difference are discussed, particularly the effect of body condition.

intake / heifer / dairy cow / breed

* Travail réalisé dans le cadre du Programme de recherche-développement des Alpes du Nord 


\section{INTRODUCTION}

La capacité d'ingestion des génisses et des vaches laitières dépend de leur format, de leur âge et de leur niveau de production (Journet et al, 1965 ; Faverdin et al, 1987; Troccon, 1987), ainsi que, dans une moindre mesure, de leur état d'engraissement (Garnsworthy et Topps, 1982). Ces relations ont été bien mises en évidence chez des animaux de type Pie-noir, mais peu de travaux ont été entrepris pour quantifier un éventuel effet de la race sur la capacité d'ingestion des animaux laitiers (Korver, 1982 ; Gruber et al, 1991). On sait pourtant que ce facteur joue un rôle important chez les animaux à viande (Petit et al, 1987). Chez les vaches laitières de race anglo-saxonnes, des coefficients de correction ont aussi été proposés pour tenir compte de cet effet (Fox et al, 1992). Dans les systèmes d'alimentation des ruminants proposés par I'INRA, la prévision des quantités ingérées est réalisée grace au système des Unités d'encombrement qui permet à la fois d'exprimer et de prévoir l'ingestibilité des aliments et la capacité d'ingestion des animaux (Jarrige et al, 1986 ; Dulphy et al, 1987). Ce système a été élaboré et validé essentiellement à partir de données recueillies sur les animaux de type Pie-Noir.

À partir des données recueillies au cours de 6 années d'expérimentation sur des animaux de race Holstein, Montbéliarde et Tarentaise, conduits ensemble et alimentés avec les mêmes fourrages au cours de leur période d'élevage et de leurs 4 premières lactations, l'objectif de cette étude a été de préciser les éventuelles différences de capacité d'ingestion entre ces 3 types d'animaux, et ainsi de tester la validité du modèle de prédiction des quantités ingérées sur une large gamme d'animaux.

\section{MATÉRIEL ET MÉTHODES}

Cette étude a été réalisée à partir des données issues de lots de 4 à 15 animaux de races Holstein, Montbéliarde et Tarentaise, conduits sur le domaine INRA de Marcenat (Cantal). Les animaux de race Holstein et Montbéliarde provenaient du troupeau de ce domaine. Les animaux de race Tarentaise provenaient de différents élevages de Savoie, Isère, Hautes-Alpes et HauteLoire. Ils avaient été introduits à l'âge de 3 mois sur le domaine de Marcenat. Les lots avaient été constitués dans le cadre d'expérimentations concernant l'étude des caractéristiques de ces 3 types d'animaux lors de leur phase d'élevage (D'Hour et al, 1991) ou de leur réaction à une sous-alimentation énergétique en période de lactation (Coulon et D'Hour, 1994).

Au cours de chaque comparaison (2 pendant la phase d'élevage, 2 chez des vaches primipares, une au cours de chacune des lactations 2 à 4), tous les animaux recevaient, à volonté, la même ration de base, composée de foin de prairie naturelle $(0,63$ à 0,71 UFL $/ \mathrm{kg}$ MS, 59 à $69 \mathrm{~g}$ PDIN/kg MS, 72 à $75 \mathrm{~g}$ PDIE/kg MS). Au cours des 3 premières lactations, cette ration était complémentée à 2 niveaux d'apports énergétiques, haut (selon les recommandations INRA, 1988) ou bas ( $3 \mathrm{~kg}$ de concentré de type production $(1,07$ UFL/kg MS, $126 \mathrm{~g}$ PDIN $/ \mathrm{kg}$ MS, $115 \mathrm{~g}$ $\mathrm{PDIE} / \mathrm{kg} \mathrm{MS}$ ) en moins par vache pour un même niveau de production attendue). D'une lactation à l'autre, les vaches étaient affectées à un des 2 niveaux de complémentation de manière aléatoire. En 4 lactation, seules des vaches Holstein et Tarentaises recevant un seul niveau d'apport de concentré (haut) ont été utilisées. Cette étude a donc été réalisée à partir des données observées sur 12 lots de génisses et 26 lots de vaches, au cours de périodes de 2 mois, situées en fin d'hiver et, pour les vaches, au moins 2 mois après leur vêlage.

Au cours de chaque comparaison, la valeur d'encombrement du fourrage a été obtenue à partir des données de quantités ingérées observées sur le ou les lots d'animaux Holstein, de la valeur énergétique des aliments (estimées pour les fourrages d'après leur digestibilité mesurée sur moutons) et de la capacité d'ingestion des animaux calculée d'après leurs performances observées durant les périodes correspondantes 
(poids vif et/ou lait à $4 \%$ de matières grasses) (Troccon, 1987 ; Faverdin et al, 1987), en appliquant les modèles proposés par Dulphy et al (1987). Ensuite, ces mêmes modèles ont été utilisés pour calculer les quantités ingérées prédites pour chacun des lots des autres types d'animaux. Les valeurs prédites par les modèles ont alors été comparées à celles réellement observées.

\section{RÉSULTATS ET DISCUSSION (fig 1)}

Au cours de la phase d'élevage, chez les génisses Montbéliardes et Tarentaises, les quantités ingérées observées ont été exactement égales à celles prédites. En lactation, l'écart entre les quantités prédites et observées a été faible et non significatif chez les vaches Montbéliardes, quel que soit leur numéro de lactation (respectivement $-0,2$ et $+0,6 \mathrm{~kg} \mathrm{MS} / \mathrm{j}$ chez les primipares et les multipares). Chez les vaches Tarentaises, cet écart a été très faible en première lactation $(+0,1 \mathrm{~kg} \mathrm{MS} / \mathrm{j})$, mais plus élevé au cours des lactations suivantes $(-1,8 \mathrm{~kg} \mathrm{MS} / \mathrm{j}$ en moyenne sur les 5 lots utilisés, $P<0,01$ ), en particulier dans les lots bas où il a atteint $-2,8 \mathrm{~kg} \mathrm{MS} / \mathrm{j}$. Cet écart plus important dans les lots Bas est dû à un taux de substitution fourrage/concentré plus faible chez les vaches Tarentaises que chez les vaches Holstein (Coulon et D'Hour, 1994).

II semblerait donc que la capacité d'ingestion des vaches Tarentaises adultes soit sensiblement inférieure (d'environ 1 UE lorsqu'elles sont alimentées selon les recommandations) à celle de vaches Holstein ou Montbéliardes conduites dans des conditions semblables. Cette différence est peutêtre en partie due à l'écart d'état corporel entre ces 2 types d'animaux $(+0,6$ point en faveur des Tarentaises), qui résulte de la mobilisation des réserves corporelles beaucoup plus importante en première lactation chez les Holstein que chez les Tarentaises (Coulon et D'Hour, 1994). Le très faible état corporel des vaches multipares Holstein en

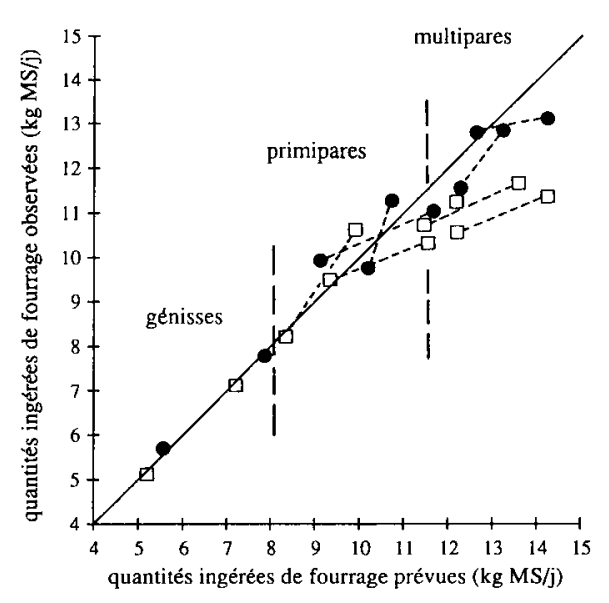

Fig 1. Relation entre les quantités ingérées de fourrage observées et prédites (à partir de celles ingérées par les animaux de race Holstein, $c f$ texte) selon l'âge, la race et le niveau de complémentation des animaux. Les 2 lots, haut et bas, d'une même comparaison intra-race sont reliés entre eux. Dans chaque comparaison, le lot $\mathrm{B}$ est celui le plus à droite. $\square$ Tarentaise ; Montbéliarde.

début de période de mesure $(1,1$ en moyenne sur les 4 lots utilisés en $2^{e}$ et $3^{e}$ lactation) a certainement contribué à accroître leur capacité d'ingestion. On sait en effet que des vaches maigres ingèrent plus, toutes choses étant égales par ailleurs, que des vaches grasses (Garnsworthy, 1988). Ceci est confirmé par les très faibles valeurs d'encombrement des fourrages que nous avons estimées à partir des quantités ingérées par ces animaux $(0,99 \mathrm{UEL} / \mathrm{kg} \mathrm{MS}$ en moyenne). Cependant, la différence d'état corporel des 2 types d'animaux n'est certainement pas suffisante pour expliquer totalement l'écart de capacité d'ingestion chez les vaches multipares. Ainsi, au cours de la dernière comparaison (en 4 e lactation), où les états corporels étaient voisins (respectivement 1,4 et 1,6 pour les Holstein 
et les Tarentaises), les vaches Tarentaises ont ingéré près de $1 \mathrm{~kg} \mathrm{MS} / \mathrm{j}$ de fourrage de moins que prévu.

Dans l'état actuel des choses, cette étude montre que les modèles de prévision de la capacité d'ingestion des génisses et des vaches laitières proposées dans le cadre des nouvelles recommandations INRA (1988) sont applicables à une large gamme d'animaux. En pratique, les différences entre les valeurs prédites et observées ne sont sensibles que chez les vaches Tarentaises adultes. L'application des modèles à ces animaux conduit, en moyenne, à une sousestimation des apports d'aliment concentré (à 1,05 UFL $/ \mathrm{kg} \mathrm{MS}$ ) d'environ $0,7 \mathrm{~kg} / \mathrm{j}$ pour une vache adulte produisant $20 \mathrm{~kg} / \mathrm{j}$ de lait, recevant une ration à base de foin de bonne qualité (1,05 UEL et 0,70 UFL/kg MS) complémentée de manière à couvrir ses besoins. Cette étude confirme par ailleurs la nécessité de poursuivre des travaux permettant de prendre en compte d'autres facteurs de variation, tels que l'état corporel des animaux.

\section{RÉFÉRENCES}

Coulon JB, D'Hour P (1994) Effet du niveau des apports énergétiques sur les performances de vaches laitières de race Holstein ou Tarentaises. Ann Zootech 43, 355-368

D'Hour P, Coulon JB, Garel JP (1991) Capacité d'ingestion des génisses : influence de la race, de l'âge et de la qualité du foin. Ann Zootech 40, 171-179
Dulphy JP, Faverdin P, Micol D, Bocquier F (1987) Révision du système des Unités d'encombrement (UE). Bull Tech CRZV Theix, INRA, 70, 35-48

Faverdin P, Hoden A, Coulon JB (1987) Recommandations alimentaires pour les vaches laitières. Bull Tech CRZV Theix, INRA, 70, 133-152

Fox DG, Sniffen CJ, O'Connor JD, Russel JB, Van Soest PJ (1992) A net carbohydrate and protein system for evaluating cattle diets: III. Cattle requirements and diet adequacy. J Anim Sci 70, 3758-3596

Garnsworthy PC, Topps JH (1982) The effect of body condition of dairy cows at calving on their food intake and performances when given complete diets. Anim Prod 35, 113-119

Garnsworthy PC (1988) The effect of energy reserves at calving on performance of dairy cows. $n$ : Nutrition and lactation in the dairy cow (PC Garnsworthy, ed), Butterworths, Londres, 157-170

Gruber L, Steinwender R, Krimberger K, Sölkner J (1991) Roughage intake of Simmental, Brown Swiss and Holstein Friesian Cows fed rations with 0,25 and $50 \%$ concentrates. Liv Prod Sci 27, 123-136

INRA (1988) Alimentation des bovins, ovins et caprins ( $R$ Jarrige, ed). INRA, Paris, $47 \mathrm{tp}$

Journet M, Poutous M, Calomiti S (1965) Appétit de la vache laitière. I Variations individuelles des quantités ingérées. Ann Zootech 14, 5-37

Jarrige R, Demarquilly C, Dulphy JP, Hoden A, Robelin $J$, Beranger $C$, Geay $Y$, Journet M, Malterre C, Micol D. Petit M (1986) The INRA 'fill unit' system for predicting the voluntary intake of forage-based diets in ruminants. A review. J Anim Sci 63, 1737-1758

Korver $S$ (1982) Feed intake and production in dairy breeds dependent on the ration, Ph D Thesis, Agr Univ Wageningen

Petit M, Garel JP, Grenet N (1987) Utilisation des foins et pailles par le troupeau de vaches allaitantes. In : Les fourrages secs : récolte, traitement, utilisation (C Demarquilly, ed), INRA, Paris 361-389

Troccon JL (1987) Recommandations alimentaires pour les veaux et génisses d'élevage. Bull Tech CRZV Theix, INRA, 70, 167-172 